\title{
Structure and Mass Function of Open Cluster NGC 6910
}

\author{
Harmeen Kaur ${ }^{1}$, Saurabh Sharma ${ }^{2}$ and Alok K. Durgapal ${ }^{1}$ \\ ${ }^{1}$ Department of Physics (UGC-Centre of Advanced Study), Kumaun University, Nainital, India. \\ ${ }^{2}$ Aryabhatta Research Institute of Observational Sciences, Manora Peak, Nainital, 263002, India \\ Email: harmeenkaur.kaur229@gmail.com
}

\begin{abstract}
NGC 6910 is located in a Cygnus $X$ region, which is a $\sim 10^{\circ}$ complex of actively star forming molecular clouds and young clusters, located at a distance of about $1.7 \mathrm{kpc}$ (Reipurth \& Schneider 2008). Open clusters possess many favorable characteristics for initial mass function (IMF) studies. The observed mass function of a star cluster can in principle be determined from the observed luminosity function (LF) using theoretical stellar evolutionary models. Here, we are presenting our initial results related to structure parameters, extinction, distance and mass function of open cluster NGC 6910 based on the deep and wide field mosaic images taken from 1.0m Sampurnand telescope of ARIES, India.
\end{abstract}

Keywords: Star clusters, Structure parameters, Mass function.

\section{Introduction}

Open clusters consist of two main regions i.e., the core (nucleus) and the corona (prolonged region of the star cluster) regions (Kholopov 1969), and they have important bearing on studies related with star formation and Galactic evolution as the higher mass stars tends to move towards core region with time due to dynamical evolution. The core of the clusters generally contains relatively bright and massive stars $\left(\geq 3 \mathrm{M}_{\odot}\right)$, whereas the corona contain large number of faint and low mass stars $\left(\leq 1 \mathrm{M}_{\odot}\right)$. Although various efforts has been made in understanding star formation processes in high mass stars forming region, but the satisfactory explanation is still far from reality. Young open clusters (age $<10 \mathrm{Myr}$ ), having just formed from gravitationally bound molecular clouds and still embedded in the parent nebulous regions, present an unique laboratory for understanding the process of star formation, its history, the early evolution of stars over a wide mass range. These regions contains stars of high mass as well as low mass pre-main sequence (PMS) stars. Broad mass range of the cluster members can also be used to quantify the relative numbers of stars in different mass bins and to constrain initial mass function (IMF) which is an important statistical tool to understand the formation of stars and brown dwarfs. Whether the IMF is universal with a shape similar to that of the Galactic field with a general flattening below $1 \mathrm{M}_{\odot}$ or is a function of star formation environment is a question yet to be answered (Bastian et al. 2010). The higher-mass stars mostly follow the Salpeter mass function (Salpeter 1955). At lower masses, the IMF is less well constrained, but appears to flatten below $1 \mathrm{M}_{\odot}$ and exhibits a turnover between 0.1 and $0.7 \mathrm{M}_{\odot}$ with fewer stars of the lowest masses (Kroupa \& Boily 2002; Chabrier 2003). While the higher-mass domain is thought to be mostly formed through fragmentation and/or accretion onto the protostellar core (e.g., Padoan \& Nordlund 
2002; Bonnell et al. 2006). Few example exist in literature (for e.g. Pandey et al. 2001, 2005) showing change in the slope of the MF as a function of radial distance from the cluster center.

\section{Observations and Data Reduction}

The CCD, UBVRI photometric data of the NGC 6910 region were acquired on 13, 15
June 2005, 04 November 2005 and 26-29 September 2006, respectively, by using the $2048 \times 2048$ pixel $^{2}$ CCD camera mounted on the $f / 13$ Cassegrain focus of the 104-cm Sampurnanand telescope of Aryabhatta Research Institute of Observational Sciences (ARIES), Nainital, India. Initial processing of

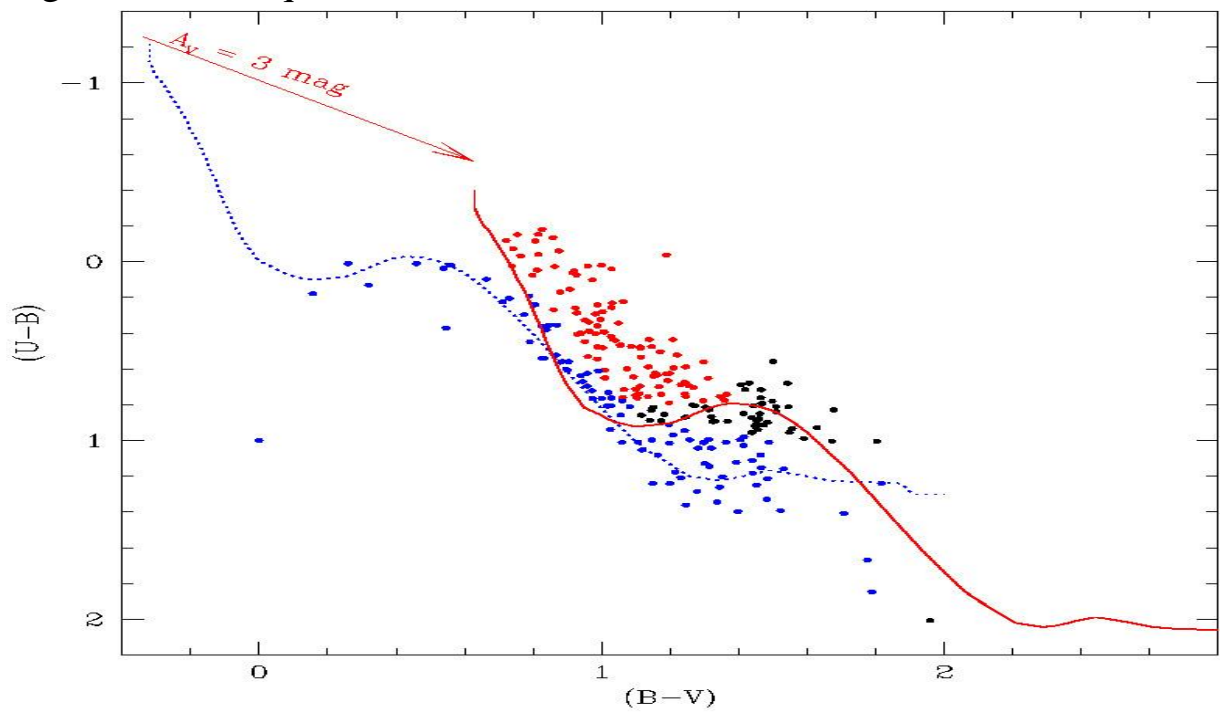

Fig.1 (Color online): $(\mathrm{U}-\mathrm{B}) /(\mathrm{B}-\mathrm{V})$ TCD for the sources in the NGC 6910 region. The dotted blue curve represents the intrinsic Zero Age Main Sequence (ZAMS) for Z $=0.02$ by Schmidt Kaler (1982). The continuous red curves represent the Schmidt-Kaler (1982). ZAMS shifted along the reddening vector of 0.72 for $\mathrm{E}(\mathrm{B}-\mathrm{V})=0.95 \mathrm{mag}$.

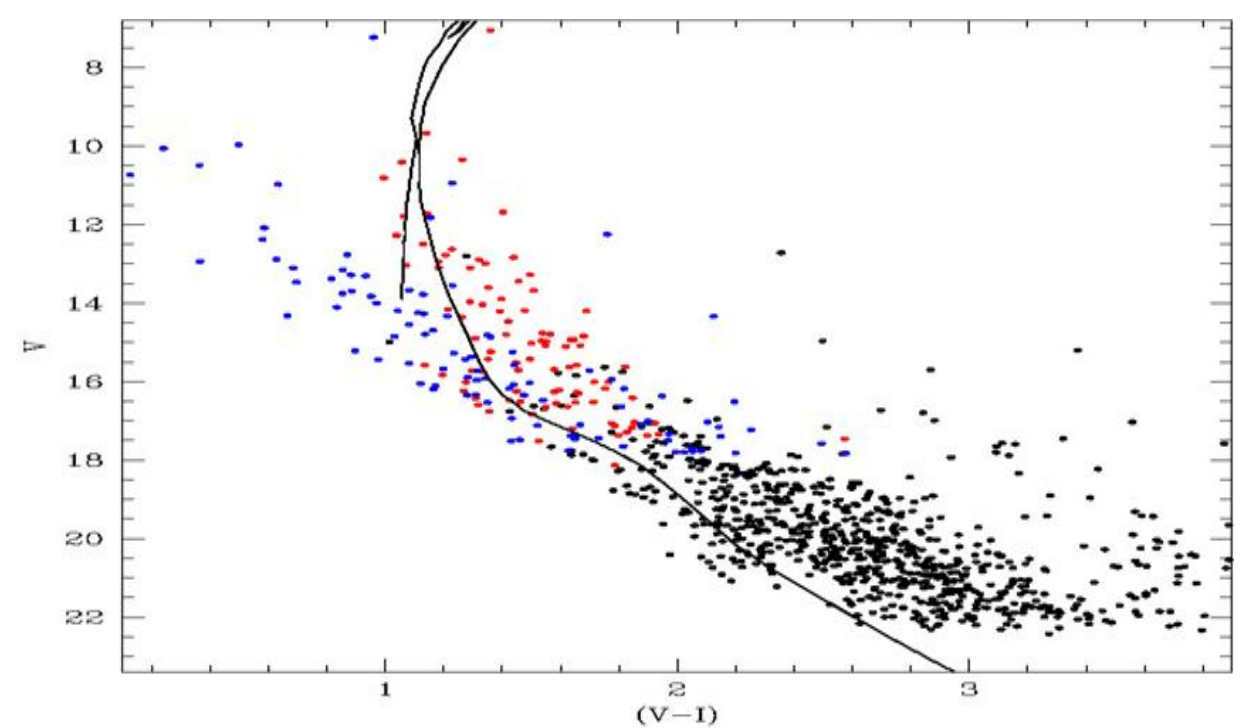

Fig.2 (Color online): $\mathrm{V} /\left(\mathrm{V}-\mathrm{I}_{\mathrm{c}}\right) \mathrm{CMD}$ for all the optically detected sources in the NGC6910 region. The isochrone for 6.65 Myr by Marigo et al. (2008) (thickblack curve) corrected for the distance and reddening of $1.82 \mathrm{kpc}$ and $\mathrm{E}(\mathrm{B}-\mathrm{V})=0.95$ mag respectively. 
the data frames was done by using the IRAF and ESO-MIDAS data reduction packages. Photometry of the cleaned frames were carried out by using DAOPHOT-II software (Stetson 1987). The point spread function (PSF) was obtained for each frame by using several uncontaminated stars. Magnitudes obtained from different frames were averaged. We have carried out a comparison of the present data with those present in the literature. The comparison indicates that the $\mathrm{V}$ magnitude and $(\mathrm{B}-\mathrm{V})$ colors obtained in the present work are in agreement with those observed in literature. In particular it is very important to know the completeness limits in terms of mass to derive MF slope. The IRAF routine ADDSTAR of DAOPHOT II was used to determine the completeness factor (CF). In this study, our data is complete upto 20.6 mag in $\mathrm{V}$ band and corresponding mass completeness limit is $0.8 \mathrm{M}$.

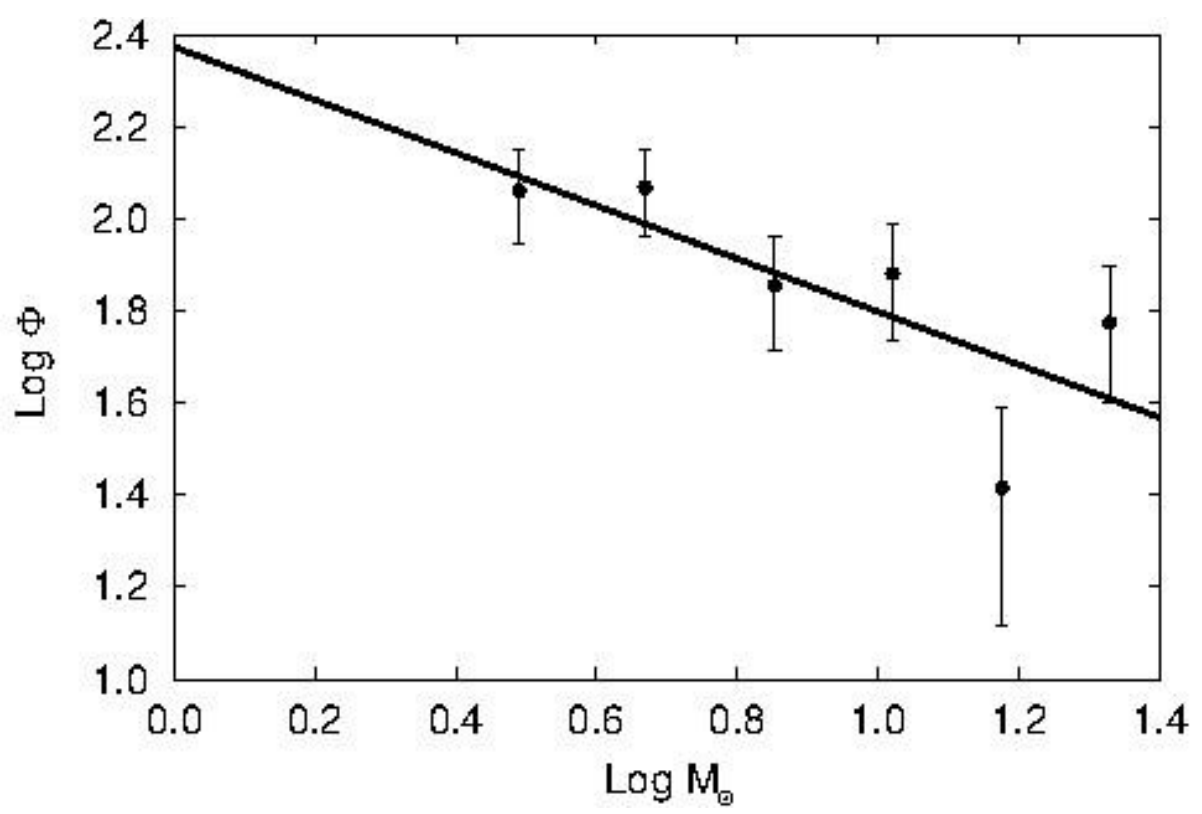

Fig.3: A plot of MF for Cluster NGC 6910 (main-sequence stars). Log $\varphi$ represents $\log$ ( dN/dlog $\mathrm{m})$. The error bars represents $\pm \mathrm{N}$ error. Continuous curves show a least-squares fit for the given mass range.

\section{Results and Analysis}

The structure and radial extent of the cluster (core and corona regions) can be studied by means of density estimations. By fitting the King-model surface-density profile to the observed radial-density profile of stars with $\mathrm{V} \simeq 19$ mag we have found that the core and cluster radius are 1.4 arcmin and 5.5 arcmin, respectively. The extinction towards the cluster NGC 6910 was estimated by using the

(U-B)/(B-V) Two Color Diagram (TCDs), shown in Fig.1 ; this gives $\mathrm{E}(\mathrm{B}-\mathrm{V})$ min $=$ 0.95 mag. By using Color- Magnitude Diagram (CMD), shown in Fig.2, we estimated the distance of NGC 6910 cluster, 
which comes out to be $1.82 \mathrm{kpc}$. In present study, with the help of statistically cleaned CMD we can derive the MF using theoretical evolutionary models. Since, the stars having $\mathrm{V} \leq 16$ mag are considered to be still on the MS, for these stars the LF was converted into MF using theoretical model by Marigo et al. (2008) (cf. Pandey et al. (2001, 2005). The resulting MF of the cluster (MS stars) is plotted in Fig.3. The slope $(\Gamma)$ of the MF in the mass range $2.3 \leq \mathrm{m} / \mathrm{M}_{\odot} \leq 24.86$ comes out to be $-0.67 \pm 0.12$, keeping the errors in the estimation of $(\Gamma)$, slope appears to be shallower than the Salpeter (1955) value (1.35).

\section{References}

Bastian, N., Covey, K. R., \& Meyer, M. R. 2010, ARA\&A, 48, 339

Bonnell, I. A., Clarke, C. J., \& Bate, M. R. 2006, MNRAS, 368, 1296

Chabrier, G. 2003, PASP, 115, 763

Kholopov, P. N. 1969, Soviet Ast., 12, 625

King, I. 1962, AJ, 67, 471

Kroupa, P., \& Boily, C. M. 2002, MNRAS, 336, 1188

Marigo, P., Girardi, L., Bressan, A., et al. 2008, A\&A, 482,883

Padoan, P., \& Nordlund,A ‥ 2002, ApJ, 576, 870

Pandey, A. K., Nilakshi, Ogura, K., Sagar, R., \& Tarusawa, K. 2001, A\&A, 374, 504

Pandey, A. K., Upadhyay, K., Ogura, K., et al. 2005, MNRAS, 358, 1290

Reipurth, B., \& Schneider, N. 2008, Star Formation and Young Clusters in Cygnus, ed. B. Reipurth, 36.

Salpeter, E. E. 1955, ApJ, 121, 161

Schmidt-Kaler, T. 1982, in LandoltB ornstein: Numerical Data and Functional
Relationship in Science and Technology, Vol. 2b. eds. Schaifers K., Voigt H. H., Landolt H. (Springer-Verlag), Berlin, p. 19, ed. V. H. H.L. H. Schaifers, K.

Stetson, P. B. 1987, PASP, 99, 191 